\title{
CRECIMIENTO ECONÓMICO EN EL ESPACIO PERUANO, 1681-1800: UNA VISIÓN A PARTIR DE LA AGRICULTURA :
}

\section{CARLOS NEWLAND}

Universidad Argentina de la Empresa

\section{JOHN COATSWORTH}

David Rockefeller Center for Latin American Studies. Universidad de Harvard

\section{RESUMEN}

El trabajo presenta un análisis agregado y por región de la evolución de la agricultura en el espacio peruano entre 1681 y 1800 , utilizando series deflactadas de diezmos. Concluye señalando que, luego de una caída inicial, existió una expansión de la agricultura durante el siglo XvIII, con una aceleración en la segunda mitad del siglo. El incremento en la producción no fue homogéneo, existiendo una destacada disparidad regional.

\section{ABSTRACT}

The article presents an aggregate and regional analysis of the evolution of agriculture in the Peruvian space between 1681 and 1800 , using deflated tithe series. It concludes that after an initial collapse, agricultural production expanded in the XVIII century, an expansion that accelerated in the second half of the century. This increase in production was not homogeneous across regions.

La evolución económica global del Espacio Peruano - siguiendo la denominación de Assadourian- ha sido estudiada por una variedad de

' Una versión inicial de este trabajo fue elaborada mientras Carlos Newland fue De Fortabat Fellow en el David Rockefeller Center for Latin American Studies de la Universidad de Harvard. Asimismo, Carlos Newland se benefició de un subsidio de investigación otorgado por la Fundación Antorchas. Los autores agradecen la colaboración recibida de John Couriel e Irina Werning. El trabajo fue presentado en la reunión de LACLIO (Latin American Cliometrics Society Conference), realizada en Cartagena en agosto de 1999. 
autores, incluyendo a Klein (1998), O'Phelan Godoy (1988), Romano (1993), Romano y Carmagnani (1999), Tandeter (1995), Slicher Van Bath (1989) y el mismo Assadourian (1983). Con referencia al período 1681-1800, analizado en este trabajo, la imagen más común es de una crisis en la primera mitad del siglo XVII - respecto a una situación más favorable a finales del XVII- y una mejora (en casos discutida) en la segunda mitad del siglo xviII. Algunos autores, como Klein ${ }^{2}$, postulan una fuerte relación entre actividad económica general y la minería; para otros, como Slicher Van Bath ${ }^{3}$, esta relación se iría debilitando a lo largo del siglo XVIII, al aparecer una estructura productiva más diversa, con mayor peso de la agricultura y el comercio. En general los estudios han señalado un proceso gradual de desconcentración regional, debida a la aparición de nuevos centros económicos que se sumaron a los tradicionales ${ }^{4}$.

Los trabajos más cuantitativos, como los de Klein y Slicher Van Bath, se han concentrado en la evolución de las cuentas del Fisco en sus respectivos rubros. En su análisis la evolución de la agricultura ha tenido una importancia menor, y no han explotado el rubro más relacionado con las actividades rurales, los novenos reales. Es verdad que muchos autores han investigado la evolución de los novenos -o lo que es lo mismo los diezmos- para regiones específicas (en general obispados), como Buenos Aires ${ }^{5}$, Chile ${ }^{6}$, Arequipa ${ }^{7}$, Cuzco $^{8}$, Cochabamba ${ }^{9}$, La Plata ${ }^{10}$, Huaman$\mathrm{ga}^{11}$ y Lima ${ }^{12}$. Sin embargo, falta aún un análisis del espacio peruano en su conjunto, aquí definido como la superficie cubierta por el Virreinato del Perú hacia $1750^{13}$. Este trabajo busca subsanar esa deficiencia, presentando series de diezmos para la región entre 1681 y 1800 . En primer lugar se estudia el comportamiento de los precios agrícolas, que luego servirán para deflactar los diezmos. Más adelante se presentan las series de diezmos en valores nominales y reales, y se estudia su evolución general

${ }^{2}$ Klein (1988), p. 35.

${ }^{3}$ Slicher Van Bath (1989), pp. 142-143.

4 Slicher Van Bath (1989), pp. 142-143; Klein (1989), p. 106.

5 Amaral y Ghio (1990), Garavaglia (1989), Guerrero Soriano (1994).

" Carmagnani (1969), Larraín (1990).

'Brown (1986), Huertas Vallejos y Carnero Albarrán (1983b).

${ }^{*}$ Huertas Vallejos y Carnero Albarrán (1983a).

${ }^{9}$ Larson (1998).

11) Quiroz Chueca (1984), Tandeter y Watchel (1990).

11 Huertas Vallejos (1982).

12 Burga (1989), Carnero Albarrán y Pinto Huaracha (1983).

13 Por tanto, se excluye el territorio incluido en el siglo xvili en el Virreinato de Nueva Granada y se incluye a la región comprendida por el Virreinato del Río de la Plata. 
y por región. Finalmente se contrasta la evolución global de la agricultura con las visiones generales sobre el comportamiento de la economía y se discuten algunas conjeturas sobre el tipo de expansión experimentado en el período.

\section{LOS PRECIOS}

Los diezmos utilizados en este artículo se encuentran en pesos corrientes de la época. Dado que el objetivo del trabajo es conocer la evolución del volumen de producción agrícola se hace necesario obtener índices de precios que permitan deflactar los diezmos. En esta sección se presentan series de precios para tres ciudades, Lima, Santiago de Chile y Potosí, elaborados respectivamente a partir de las recopilaciones de Macera (1992), Larraín (1990) y Tandeter y Watchel (1992). Con esta información se calculan series de precios agrícolas, luego utilizados para efectuar las correcciones a los valores nominales.

Aunque existen más series de precios para la región que las aquí utilizadas, se han seleccionado las correspondientes a Lima, Santiago y Potosí por las siguientes razones: 1) la importancia relativa de estas ciudades; 2) por presentar series largas, que cubren todo el período considerado, y 3) los precios tienen la ventaja de originarse en una fuente común y homogénea, libros de cuentas de instituciones religiosas, tales como conventos, monasterios, seminarios, colegios y hospitales. Estos precios no pueden considerarse ni mayoristas ni minoristas, situándose en algún punto medio. La razón es que por el volumen de compras estas instituciones debían obtener precios más bajos que los ofrecidos por los comercios minoristas, sin llegar a ser sin embargo precios mayoristas.

Uno de los problemas más graves al construir un índice de precios es obtener ponderaciones que se correspondan con el objeto a deflactar. Dado el objetivo de este trabajo, lo ideal hubiera sido obtener la estructura de la producción global y regional del espacio peruano para algún lapso, que brindara una idea de la importancia relativa de cada producto agrícola. Dado que no se cuenta con esta estructura, se decidió elaborar las series de cada ciudad dando idéntica ponderación a cinco productos agrícolas, que han sido, para Lima: maiz, trigo, papa, vino y azúcar; para Santiago: vino, azúcar, papa, harina y grasa; para Potosí: chuño, papa, maíz, vino y azúcar. La selección de los productos se basó tanto en alguna percepción de su importancia, como en la existencia de series más o menos completas. En el gráfico 1 


\section{GRÁFICO 1}

Evolución de los precios agricolas en el espacio peruano

$1681-1700=100$

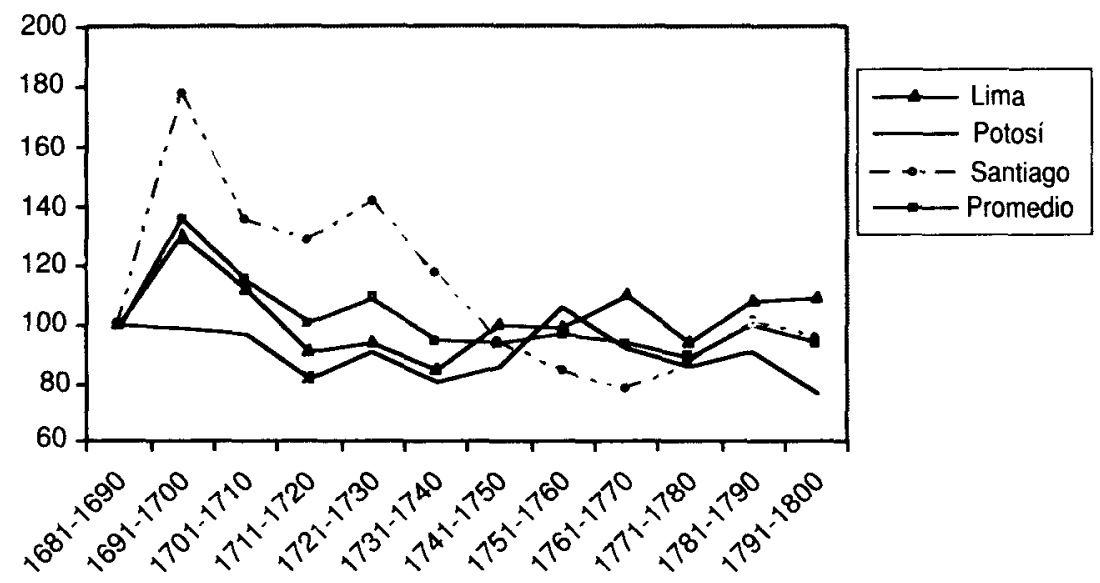

FuENTES: Apéndices.

se presentan los índices obtenidos que, dadas las limitaciones con que se han construido, no pretenden sino ser una mera aproximación a la evolución de los precios agrícolas de la región. El comportamiento del índice promedio muestra un aumento de precios en la última década del siglo XVII, para mostrar luego una tendencia decreciente hasta 1731-1740. A partir de ese momento se presenta una cierta estabilidad, hasta que se produce un leve aumento a finales de siglo. Debe mencionarse que esta tendencia general no refleja las variaciones diversas y propias de cada ciudad o región. Por ejemplo, el aumento inicial de precios en Santiago es muy agudo, al tiempo que en Potosí en esos años hay estabilidad. Finalmente debe aclararse que los índices obtenidos no son índices de costo de vida, pues no incorporan bienes manufacturados como los productos importados, o lo que es más importante, los textiles americanos. Dado que los precios de estos dos rubros presentan una caída secular durante el siglo XVIII, un índice de precios general mostraría una mayor tendencia a la baja que la que muestra un indice exclusivamente agrícola. 


\section{DIEZMOS Y PRODUCCIÓN AGRARIA}

El diezmo es uno de los indicadores de actividad económica que pueden derivarse de las cuentas fiscales producidas en todo el Imperio español. El diezmo -destinado primordialmente a costear los gastos de la Iglesiacomprendía en general una décima parte de la producción rural, incluyendo a cereales, vino, azúcar y ganado. Era aplicado principalmente a la producción de haciendas, aunque también afectaba a la producción indígena si la misma estaba destinada al mercado ${ }^{14}$. La razón de su aparición en las cuentas fiscales es que una novena parte de esta contribución correspondía al Estado. De todos los indicadores de actividad económica derivados de las cuentas fiscales el diezmo parece el más confiable, pues su

\section{CUADRO 1 \\ Diezmos del Virreinato del Perú deflactados $y$ con base $1681-1700=100$}

\begin{tabular}{|c|c|c|c|c|c|c|c|c|c|c|}
\hline & Trijillo & Lima & Cuzco & Arequipa & La Paz & La Plata & Cbile & $\begin{array}{c}\text { Buenos } \\
\text { Aires }\end{array}$ & Total & $\begin{array}{c}\text { Total } \\
(\text { sin } \\
\text { Lima) }\end{array}$ \\
\hline $1681-1690 \ldots \ldots \ldots$ & 100 & 100 & 100 & 100 & 100 & 100 & 100 & 100 & 100 & 100 \\
\hline $1691-1700 \ldots \ldots \ldots$ & 91 & 42 & 93 & 79 & 78 & 108 & 81 & 82 & 58 & 82 \\
\hline $1701-1710 \ldots \ldots \ldots$ & 87 & 66 & 94 & 99 & 76 & 102 & 168 & 139 & 78 & 97 \\
\hline $1711-1720 \ldots \ldots \ldots$ & 172 & 40 & 123 & 115 & 105 & 106 & 170 & 119 & 68 & 114 \\
\hline $1721-1730 \ldots \ldots \ldots$ & 115 & 53 & 133 & 108 & 121 & 80 & 214 & 129 & 72 & 109 \\
\hline $1731-1740 \ldots$ & 102 & 56 & 147 & 163 & 143 & 90 & 196 & 172 & 80 & 122 \\
\hline $1741-1750 \ldots \ldots \ldots$ & 84 & 42 & 100 & 181 & 121 & 118 & 262 & 315 & 83 & 136 \\
\hline $1751-1760 \ldots \ldots \ldots$ & 101 & 40 & 244 & 191 & 118 & 113 & 405 & 641 & 98 & 179 \\
\hline $1761-1770 \ldots \ldots \ldots$ & 121 & 35 & 120 & 296 & 119 & 128 & 466 & 535 & 100 & 185 \\
\hline $1771-1780 \ldots \ldots \ldots$ & 178 & 40 & 182 & 291 & 174 & 150 & 571 & 670 & 119 & 228 \\
\hline $1781-1790 \ldots \ldots \ldots$ & 227 & 39 & 183 & 310 & 155 & 176 & 611 & 764 & 127 & 247 \\
\hline $1791-1800 \ldots \ldots \ldots$ & 241 & 45 & 160 & 353 & 140 & 194 & 587 & 1.052 & 136 & 255 \\
\hline
\end{tabular}

Fuentes: Apéndice. Los diezmos de Trujillo, Lima y Cuzco se deflactaron con el indice de precios de Lima. Los de Chile con los de Santiago y los de La Plata con los de Potosí. Los de La Paz con el promedio de Lima y Potosí. Los de Buenos Aires con Arequipa y los totales con los promedios generales.

it La producción indigena de granos europeos para el mercado pagaba el 5 por 100; al vino se aplicaba una tasa algo menor al 10 por 100 . 
tasa se mantuvo constante en el tiempo, a diferencia de los impuestos a la actividad minera, al comercio o el tributo indigena. Por otra parte su recaudación generalmente se subastaba en remates abiertos por un organismo que incluía representantes de la Iglesia y el Estado. Esta representación múltiple, y en especial la participación de la Iglesia, fueron una garantía de control de la corrupción y del carácter competitivo de las subastas, dado que existía un interés directo en la recaudación.

Como indicador el diezmo presenta algunos problemas. En primer lugar no es un reflejo directo de la producción agropecuaria, sino una estimación de mediano plazo de la producción por parte de los recaudadores (descontando su ganancia y gastos). Por ende, los diezmos no pueden ser utilizados para analizar los cambios anuales en la producción agrícola, a excepción de los contados casos en que fueron administrados directamente por la Iglesia o el Estado. Esto no es un problema para este trabajo, que se concentra únicamente en las tendencias de largo plazo. También se ha criticado el uso de la recaudación diezmal como indicador de producción, por no considerar variaciones en los precios, crítica no aplicable a este trabajo por efectuarse los ajustes correspondientes. Finalmente debe advertirse que las series aquí presentadas no incluyen una parte muy importante de la producción agrícola, aquella producida por la población indígena para su autoconsumo. Si la producción indígena se comportó de manera muy distinta a la producción comerciada, entonces parte de las conclusiones de este trabajo se verían debilitadas ${ }^{15}$.

En el gráfico 2 se presentan series de diezmos para el total del Virreinato de Perú (en su superficie de 1750) para el período 1681-1800. En buena medida la fuente del cuadro está derivada de los novenos cobrados por el Fisco, recopilados por TePaske y Klein (1982). En los casos en que hay estudios particulares que cubren todo el lapso, como es el caso de Chile, Arequipa, La Plata y, parcialmente, Lima y Buenos Aires, se ha preferido esta fuente. Se han presentado series sólo para aquellos distritos diezmales para los que había datos relativamente completos, con lo que han quedado fuera Huamanga, Santa Cruz, Tucumán y Paraguay. Los datos anuales presentan deficiencias, pues en algunos casos faltan, y en otros hay cifras muy altas para un año dado, indicio de que posiblemente sean pagos de deudas atrasadas de los arrendatarios de diezmos. Por esta razón se han tomado promedios por década de los años en que había información (siempre que hubiera datos para al menos dos años).

15 La producción indígena para el mercado en Charcas parece haber seguido una tendencia similar a la producción no indigena. Véase Tandeter (1995), p. 16. 


\section{GRÁFICO 2}

Espacio peruano y Lima. Evolución de la agricultura 1681-1800

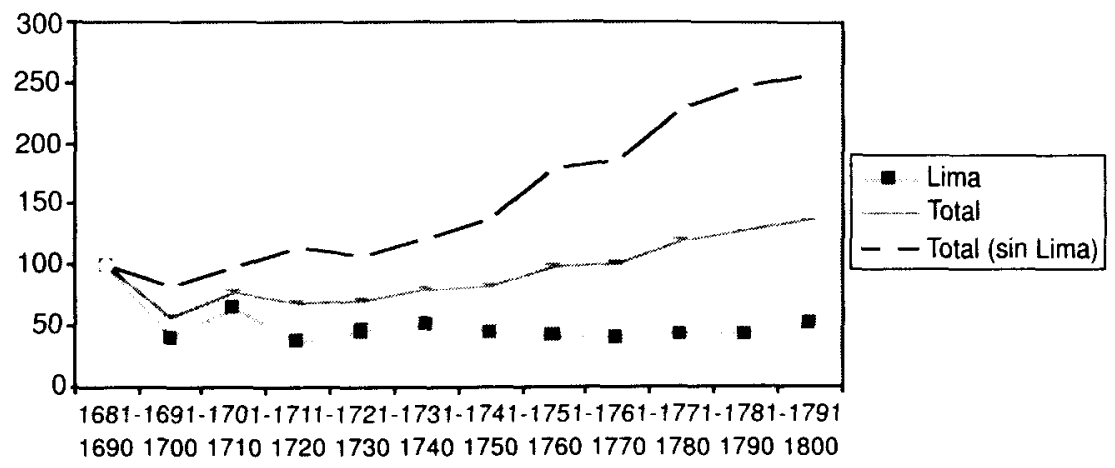

FUENTES: Apéndice.

Cabe preguntarse cuán representativas eran estas cifras de la masa decimal total del espacio peruano. Dicho ejercicio es posible para mediados del siglo XVIII ya que existe una estimación independiente preparada por el funcionario Miguel Feijoo de Sosa para el Virrey Manuel Amat. Contrastando el promedio anual de los diezmos utilizados en este trabajo para el período 1745-1755 (492.200 pesos) con la estimación de Feijoo para 1750 (532.200 pesos) surge una cobertura muy amplia, de más del 92 por 100 . Es decir, que aunque en este trabajo no se incluyen las cifras que corresponden a algunos obispados, se ha captado la mayor parte de la producción agrícola diezmada ${ }^{16}$.

Los diezmos totales deflactados para el conjunto del espacio peruano muestran una caída muy aguda de la producción agrícola desde sus niveles iniciales, depresión que se prolonga hasta 1730 . A partir de entonces hay un crecimiento paulatino que continúa hasta finales del siglo XviII. Sin embargo, sólo se logra una recuperación de los niveles iniciales hacia la década de 1750 . La crisis se explica en buena medida por la caída de producción en el distrito de Lima. Si se construye una serie de los totales sin Lima, continúa existiendo una caída de la producción en la última

16. La estimación de Feijoo de Sosa se tomó de Huertas Vallejos y Carnero Albarrán (1983), p. 60. En el caso de Chile los datos son para el período 1741-1761. 
década del siglo xvi, pero es menos acentuada, presentándose inmediatamente una recuperación y expansión posterior.

\section{LA PRODUCCIÓN AGRÍCOLA POR REGIÓN}

\section{a) Lima}

La región de Lima tuvo el peor comportamiento económico de todo el espacio peruano entre 1681 y 1800 . Su producción agrícola, predominantemente de trigo y maíz, cayó drásticamente a menos de la mitad de su nivel inicial antes del fin del siglo xvI. En ese nivel estancado se mantuvo a lo largo del siglo xviII. Pueden enumerarse varias razones para explicar este fenómeno. Por el lado de la demanda la caída inicial de la producción minera y de los ingresos públicos afectó adversamente el gasto de la ciudad y de la región. A fines del siglo xvi más de la mitad de los recursos fiscales de la Caja de Lima se originaban en remisiones de otras regiones, especialmente las mineras, entre las cuales destacaba Potosí ${ }^{17}$. Estas transferencias disminuyeron dramáticamente para principios del siglo xvil debido a la crisis minera y al desvío de fondos hacia Buenos Aires. En segundo lugar, una parte de la actividad económica de Lima se sostenía gracias a su intermediación obligada del comercio internacional, intermediación que se debilitó después de 1700. Por el lado de la oferta deben mencionarse los seismos ocurridos en la región, tanto en 1687 como en 1746. El terremoto de 1687 parece haber tenido consecuencias especialmente dañinas sobre la agricultura al volver improductiva parte de la tierra. Ello ocurrió por la salinización del área cercana a la costa y por deterioro de los sistemas de regadío en los valles. A esto se agregó un empeoramiento del clima, específicamente del régimen de lluvias ${ }^{18}$. El cultivo más afectado fue el trigo, que durante todo el siglo xvul debió importarse de Chile, al disminuir sensiblemente la producción local. Por su decadencia y estancamiento la importancia relativa de la región en cuanto a la producción agrícola del espacio peruano fue reduciéndose. De recaudar al principio del período una enorme proporción de la masa decimal (según nuestras cifras, el 59 por 100), pasó al 18 por 100 a finales del siglo XViII.

\footnotetext{
17 Andrien (1985), pp. 201, 62.

1* Vega de Cáceres (1996), pp. 67 y 69.
} 


\section{b) La Plata, La Paz, Cuzco, Trujillo}

Las cuatro regiones que reúne esta sección, con una producción agrícola concentrada en trigo, maíz, papa, coca y azúcar (esta última en Cuzco y Trujillo), tienen en común ocupar un lugar intermedio entre la región más deprimida, Lima, y las regiones más dinámicas, tratadas en la próxima sección. La región de La Plata sorprende, pues podría esperarse una situación similar o peor a la de Lima, dada la caída de la actividad minera de Potosí. Sin embargo, su agricultura se mantiene en un nivel relativamente estancado (con una caída entre 1721 y 1740), recuperándose a mediados de siglo y mostrando una cierta expansión hasta finales del siglo XvIII. El distrito de La Paz y el de Cuzco sufren una caída inicial de la que se recuperan a principios del siglo XviI, para mostrar un leve aumento después. Trujillo sufre un estancamiento hasta mediados del siglo XVII, para aumentar luego sensiblemente su producción. La región de Huamanga, para la que sólo existen datos parciales, también muestra crecimiento en el largo plazo ${ }^{19}$.

\section{c) Arequipa, Chile, Buenos Aires}

Arequipa, Chile y Buenos Aires tienen en común el mostrar el más acentuado crecimiento del espacio peruano. Cada una de las regiones tuvo su producto agrícola de exportación predominante, en el caso de Arequipa el vino, de Chile el trigo y en Buenos Aires el cuero y sebo. Arequipa basaría su expansión - que se nota después de 1710 - en la producción de vino y aguardiente, destinados al área central de los Andes. Su dinamismo se evidencia en que a fines del siglo xvm la producción de la región representaba el 35 por 100 de las importaciones de Potosí ${ }^{20}$. El crecimiento casi continuo de Chile fue impulsado por sus exportaciones de trigo a Lima, un hecho notable dado el costo del flete del grano. Este aumento se dio en el marco de una caída de precio del trigo, por lo que debe haber existido un importante incremento de productividad. El caso de Buenos Aires es algo distinto, pues su punto de partida es muy bajo y el incremento de la producción muy notable. La producción agrícola respondió a la importancia creciente de la región y de la ciudad de Buenos Aires, que terminó siendo cabeza del Virreinato creado en 1776. Por otra parte

\footnotetext{
${ }^{19}$ Huertas Vallejos (1982), p. 222.

21) Tandeter et. al. (1995), p. 205.
} 
en el siglo xvir continuó la cría de mulas para el Alto Perú y creció la cria de vacunos, de los que se exportaba a Europa cueros y sebo ${ }^{21}$.

\section{AGRICULTURA, CRECIMIENTO Y POBLACIÓN}

Las series sobre producción agrícola obtenidas a través de los novenos y diezmos permiten contrastar algunas de las caracterizaciones que se han hecho sobre la evolución del espacio peruano en el lapso 1681-1800:

1. Crisis inicial: Después de la década de 1680 de producción relativamente elevada, la producción agrícola cae notablemente en muchos distritos, destacando especialmente Lima. El volumen de producción para toda la región cae inicialmente en un 40 por 100 aproximadamente, y la recuperación de los niveles iniciales sólo se logra hacia 1750. Si se efectúa un cálculo global sin Lima, la crisis persiste, pero es menos aguda y la recuperación mucho más rápida. En parte esta crisis fue causada tanto por la reducción de la producción minera, como por factores de oferta.

2. Expansión económica: Si el análisis numérico se limita al siglo XvIII, las cifras muestran una tasa de incremento relativamente digna, de 0,7 por 100 anual (véase cuadro 2). Es decir, el lapso fue de un paulatino aumento de la producción, luego de una caída inicial. Este comportamiento es parecido al experimentado por la producción minera, aunque ésta parece haber crecido a una tasa superior de 1,3 por $100^{22}$. Las series permiten confirmar la idea de una progresiva aceleración económica. Durante la primera mitad del siglo el crecimiento agrícola se acerca al 0,5 por 100 anual, en la segunda mitad es mayor, de 0,9 por 100 . Debe notarse que en las regiones que presentan mayor incremento (Arequipa, Chile, Buenos Aires) no se cumple la aceleración para la segunda mitad del siglo Xvir.

3. Desconcentración regional: La producción agrícola confirma también una desconcentración económica fruto de distintas tasas de incremento regionales. Se pueden hacer tres grandes divisiones geográficas (véase cuadro 2): a) la producción en Lima decrece al 0,3 por 100 anual; b) la producción de una gran parte del área andina (Trujillo, Cuzco, La Paz y La Plata) crece a una tasa similar de 0,6 a 0,7 por $100 ; c$ ) en Arequipa, Chile y Buenos Aires el incremento es muy superior, de 1,4 a 2,5 por 100. Dado

${ }^{21}$ Sobre la economía rioplatense en el siglo xvIII véase Garavaglia (1989).

${ }^{22}$ Este crecimiento surge de una estimación del valor de las acuñaciones de oro y plata de las Casas de Moneda de Potosí, Lima y Santiago de Chile, elaboradas sobre la base de Lazo García (1992) y Toribio Medina (1902). 


\section{CUADRO 2}

Tasa anual de crecimiento agricola durante el siglo XVIII

\begin{tabular}{|c|c|c|c|}
\hline & $\begin{array}{c}1701-1720 \\
a 1741-1760\end{array}$ & $\begin{array}{c}1741-1760 \\
a 1781-1800\end{array}$ & $\begin{array}{c}1701-1720 \\
\text { a } 1781-1800\end{array}$ \\
\hline 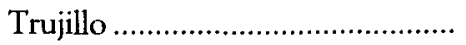 & $-0,8$ & 2,3 & 0,7 \\
\hline 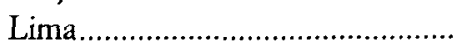 & $-0,6$ & 0,1 & $-0,3$ \\
\hline Cuzco............................. & 1,2 & 0,0 & 0,6 \\
\hline  & 0,7 & 0,5 & 0,6 \\
\hline 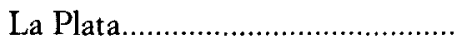 & 0,3 & 1,2 & 0,7 \\
\hline 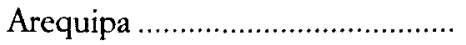 & 1,4 & 1,5 & 1,4 \\
\hline 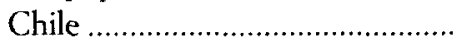 & 1,7 & 1,5 & 1,6 \\
\hline Buenos Aires.................................. & 3,3 & 1,6 & 2,5 \\
\hline 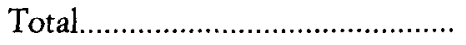 & 0,5 & 0,9 & 0,7 \\
\hline 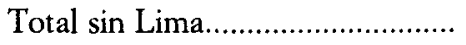 & 1,0 & 1,2 & 1,1 \\
\hline
\end{tabular}

FUENTE: Cuadro 1.

que regiones inicialmente pequeñas son las que más crecen la tendencia es a la equiparación. Un índice de Herfindahl de concentración de los diezmos (concentración total $=1$, atomización $=0$ ) pasa de un valor 0,38 en 1681-1690 a 0,15 en 1791-1800. En la urbanización también se nota la desconcentración: en 1700 sólo había dos ciudades de más de 20.000 habitantes, Lima y Potosí. Mientras que la población en estos dos centros urbanos se estancó durante el siglo xvili, otras ciudades crecieron sensiblemente superando los 20.000 habitantes para 1800, como Arequipa, Cuzco, La Paz, Santiago de Chile y Buenos Aires. La minería igualmente se descongestionó: el peso inicial predominante de Potosí se fue reduciendo a lo largo del siglo xviII ante la aparición o desarrollo de otros centros mineros en el Bajo Perú y en Chile.

4. Crecimiento per cápita: El incremento de la producción agrícola per cápita puede implicar tanto un aumento del ingreso per cápita, como un cambio estructural en la economía desde actividades de subsistencia hacia actividades basadas en el mercado. Lamentablemente no existen estimaciones confiables sobre la población del espacio peruano hacia 1700 (hacia 1800 rondarían los tres millones de habitantes), por lo que se hace difícil estimar la evolución de magnitudes económicas por habitante. Actualmente existe un consenso de que la población total se incrementó 
en el siglo xvill, pero el problema es determinar a qué tasa. Una estimación, que puede considerarse máxima, es la de Tandeter $^{23}$, quien conjetura que la población indígena de la región andina creció a una tasa anual de 1,2 por 100 durante el siglo xviII. Si esto fuera así estaríamos frente a una disminución de la producción agrícola comercial per cápita, pues la producción estaba creciendo al 0,7 por 100 anual. El mismo Tandeter ${ }^{24}$ afirma que aunque la producción de la región creció en el siglo xviI, en términos per cápita disminuyó, por lo que sostiene la probable existencia una crisis general. Otra posibilidad, preferida por los autores de este trabajo, es que la población creció de un 0,7 a 0,8 por 100 , con lo que la producción per cápita se mantendría constante ${ }^{25}$. Este aumento poblacional sería similar al experimentado por Nueva España en la misma época. Esta hipótesis de estabilidad económica -o inclusive mejoraen términos per cápita puede testarse analizando la información de la evolución de los salarios reales para la región. La idea es que una situación de involución económica se reflejaría en una caída en los salarios. La escasa información existente sobre remuneraciones laborales sugiere una cierta estabilidad en los valores nominales a lo largo del siglo xviI. En Chile, Arequipa y Potosí las retribuciones laborales no parecen haber variado sensiblemente; en Trujillo y Cuzco se incrementaron y en Lima cayeron. Dada la disminución en el nivel general de precios ocurrida a lo largo del siglo, lo más probable es que haya existido un aumento en los salarios reales para la región, con excepción de Lima ${ }^{26}$.

${ }^{23}$ Tandeter (1995), pp. 12 y 17.

${ }^{24}$ Tandeter (1995), p. 22.

${ }^{25}$ Se estimó el crecimiento para el espacio peruano con los siguientes supuestos: en Charcas la población creció al 0,8 por 100 , en Perú al 0,5 por 100 , en Chile al 1,3 por 100 y en el Río de la Plata y Tucumán al 1,3 por 100. Estas tasas muy hipotéticas están basadas en cifras de población de las siguientes fuentes: de Ramón (1992), p. 183; Loza (1949), pp. 96 y 111; Mörner (1978), p. 21; Frías (1999), p. 117; Larson (1998), p. 393. Ponderando por la población que presenta Rodríguez (1998, p. 8) para toda la región hacia 1800 (23 por 100 para Charcas, 42 por 100 para Perú, 17,5 por 100 para Chile y 17,5 por 100 para Río de la Plata), daría un crecimiento de la población de 0,8 por 100 anual para el espacio peruano. Si se calcularan los ponderadores de 1700 según estas tasas de crecimiento de población (que serían 23 por 100 para Charcas, 56 por 100 para Perú, 10,5 por 100 para Chile y 10,5 por 100 para Río de la Plata) el crecimiento sería de 0,7 por 100. Günter Vollmer, que ha estudiado la evolución demográfica del Perú colonial, ha destacado la dificultad general de reconstruir series de población. Sus estimaciones sobre la evolución de la población indigena concuerdan con la idea de un muy bajo crecimiento poblacional de los indígenas durante el siglo xvill (Vollmer, 1967, pp. 362-363 y 367-368).

${ }^{26} \mathrm{La}$ información sobre salarios se tomó de Carmagnani (1963), pp. 81-83; Brown 
Existe aún un conocimiento imperfecto del desempeño de la economía del espacio peruano durante el período colonial. Las series disponibles sobre minería, impuestos y gasto estatal han sido los indicadores más comúnmente utilizados. Las series de diezmos presentadas en este trabajo aportan información relevante sobre un sector de la economía descuidado en los análisis globales, la agricultura. Luego de un colapso a fines del siglo XVII, se ha ofrecido un cuadro ligeramente favorable sobre la evolución durante el siglo xvill. Las conclusiones son, sin embargo, provisorias, dado que la falta de series de población hace difícil estimar magnitudes per cápita.

\section{BIBLIOGRAFÍA}

Amaral, Samuel, y GHio, José María (1990), «Diezmos y producción agraria. Buenos Aires, 1750-1800», Revista de Historia Económica, VIII (3), pp. 619-647. Assadourlan, Carlos Sempat (1983), El Sistema de la Economia Colonial. El mercado interior. Regiones y Espacio Económico, México, Editorial Nueva Imagen.

ANDrien, Kenneth (1985), Crisis and Decline. The Viceroyalty of Peru in the Seventeenth Century, Albuquerque, University of New Mexico Press.

Avella Chafer, Francisco (1981), «La situación económica del clero secular de Buenos Aires durante los siglos XVII y xviII (segunda parte)», Investigaciones y Ensayos, núm. 30, pp. 283-310.

BROWN, Kendall W. (1986), Bourbons and Brandy. Imperial Reform in Eighteenth-Century Arequipa, Albuquerque, University of New Mexico Press.

Burgia, Manuel (1989), «El Perú Central, 1770-1860: Disparidades regionales y la primera crisis agricola republicana», en REINHIARD LIEHR (ed.), América latina en la época de Simón Bolivar. La formación de las economias nacionales y los intereses económicos europeos, 1800-1850, Berlín, Colloquium Verlag, pp. 227-310.

Carmagnani, Marcello (1963), El asalariado minero en Chile colonial, Santiago, Universidad de Chile.

Carmagnani, Marcello (1969), «La Producción Agropecuaria Chilena. Aspectos Cuantitativos (1680-1830)», Cabiers des Ameriques Latines, núm. 3, pp. 3-21.

Carnero Albarran, Nadia, y Pinto Huaracha, Miguel (1983), Diezmos de Lima, 1592-1858, Lima.

Escandell-Tur, Neus (1996), Producción y comercio de tejidos coloniales. Los obrajes $y$ chorrillos de Cusco 1570-1820, Cuzco, CBC.

Frias, Susana (1999), «La expansión de la población», en ACADEmia Nacionai DE LA Historia, Nueva Historia de la Nación Argentina, Buenos Aires, Planeta, II, pp. 89-126.

(1986), p. 48; Tandeter y Watchel (1992), pp. 273-276; Ramirez (1986), p. 218; Escandell-Tur (1996), p. 406; Macera (1992), i, p. xxiv. 
Garavaglia, Juan Carlos (1989), «Producción cerealera y producción ganadera en la campaña porteña: 1720-1820», en J. C. Garavaglia y Jorge Gelman, El mundo rioplatense a fines de la época colonial: estudios sobre producción y mano de obra, Buenos Aires, Editorial Biblos.

Guerrefo Sorjano, Cándido P. (1994), «Producción, evolución económica y análisis decimal. Un estudio sobre el Río de la Plata en el siglo xvilı», Anuario de Estudios Americanos, núm. 1, pp. 91-122.

Huertas VAllejos, Lorenzo (1982), «Diezmos en Huamanga», Allpancbis, núm. 20, pp. 209-235.

Huertas Vallejos, Lorenzo, y Carnero Albarrán, Nadia (1983a), Diezmos de Arequipa 1780-1856, Lima, Seminario de Historia Rural Andina.

- (1983b), Diezmos del Cuzco, 1777-1853, Lima, Seminario de Historia Rural Andina.

Johnson, Lyman L., y TANDETER, Enrique, compiladores (1992), Economias coloniales. Precios y salarios en América Latina, siglo XVIII, Buenos Aires, Fondo de Cultura Económica.

KLEIN, Herbert S. (1998), The American Finances of the Spanish Empire. Royal Income and Expenditures in Colonial México, Peri, and Bolivia, 1680-1809, Albuquerque, University of New Mexico Press.

Larraín, José (1990), «Gross National Product and Prices: The Chilean Case in the Seventeenth and Eighteenth Centuries», en JoHnSON y TANDETER (1990), pp. 109-136,

LARSON, Brooke (1998), Cochabamba, 1550-1900. Colonialism and Agrarian Transformation in Bolivia, Durham, Duke University Press.

Lazo Garcia, Carlos (1992), Economia Colonial y Régimen Monetario, 3 vols., Lima, Banco Central de la Reserva del Perú.

LozA, José Eduardo (1949), Historia de La Paz, La Paz.

Macera, Pablo (1992), Los precios del Peri. Siglos XVI-XIX. Fuentes, 3 vols., Banco Central de la Reserva del Perú, Fondo Editorial.

O'Prielan Godoy, Scarlett (1988), Perí y Bolivia, 1700-1783. Un siglo de rebeliones anticoloniales, Cuzco, Centro de Estudios Rurales Andinos Bartolomé de las Casas.

Morner, Magnus (1978), Perfil de la sociedad rural del Cuzco a fines de la colonia, Lima, Universidad del Pacífico.

Quiroz Chueca, Francisco (1984), Diezmos del Alto Peru. Chuquisaca (ss. XVII-XIX), Seminario de Historia Rural Andina.

Ramírez, Susan E. (1986), Provincial Patriarchs. Land Tenure and the Economics of Power in Colonial Peri, Albuquerque, University of New Mexico Press.

Ramón, Armando de (1992), Santiago de Cbile (1541-1991). Historia de una sociedad urbana, Madrid, MAPFRE.

Rodríguez O., Jaime (1998), The Independence of Spanish America, Cambridge, Cambridge University Press.

Romano, Ruggiero (1993), Coyunturas opuestas. La crisis del siglo xvII en Europa e Hispanoamérica, México, Fondo de Cultura Económica.

Romano, Ruggiero, y Carmagnani, Marcello (1999), «Componentes Económicos», en Ruggiero Romano, Alicia Hernandez Chávez y Marcello Carmagnani 
(coord.), Para una bistoria de América, I. Las estructuras, El Colegio de México, Feidecomiso Historia de las Américas, México, Fondo de Cultura Económica, pp. 160-287.

Slicher vaN BATH, B. H. (1989), Real Hacienda y economía en Hispanoamérica, 1541-1820, Amsterdam, CEDLA.

TANDETER, Enrique (1995), «Población y Economía en los Andes (siglo xviI), Revista Andina, núm. 1, pp. 7-22.

Tandeter, Enrique; Milletich, Vilma; Olluer, María Matilde, y Ruibal, Beatriz (1995), «Indians in Late Colonial Markets: Sources and Numbers», en Brooke Larson y Olivia Harris, con Enrique TANDETER, Ethnicity, Markets, and Migration in the Andes. At the Crossroads of History and Antbropology, Durham, Duke University Press, pp. 196-223.

TANDETER, Enrique, y WATCHEL, Nathan (1992), «Precios y producción agraria. Potosí y Charcas en el siglo Xvili», en Johnson y TANDETER (1992), pp. 221-301. TEPASKE, John Jay, y KLEIN, Herbert (1982), The Royal Treasures of the Spanish Empire in America, 3 vols., Durham, Duke University Press.

Toribio Medina, José (1902), Las Monedas Chilenas, Santiago de Chile.

VEGA DE CÁCERES, Ileana (1996), Economia Rural y Estructura Social en las Haciendas de Lima durante el siglo XVII, Lima, Fondo Editorial-Pontificia Universidad Católica del Perú.

VOLLMER, Günter (1967), Bevolkerungspolitik und Bevolkerungsstruktur der Vizekönigreich Peru zu Ende der Kolonialzeit, 1741-1821, Berlín. 


\section{APÉNDICE}

A. Evolución de los precios en el espacio peruano. Índices $1681-1690=$ base 100

\begin{tabular}{|c|c|c|c|c|}
\hline & Limo & Potosi & Santiago & Promedio \\
\hline $1681-1690$ & 100 & 100 & 100 & 99 \\
\hline 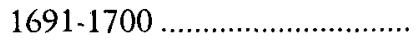 & 130 & 99 & 178 & 136 \\
\hline 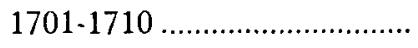 & 112 & 97 & 136 & 115 \\
\hline 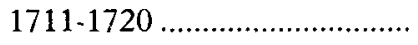 & 91 & 82 & 128 & 100 \\
\hline 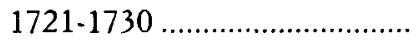 & 94 & 91 & 142 & 109 \\
\hline 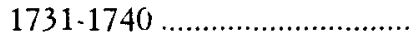 & 85 & 81 & 118 & 95 \\
\hline 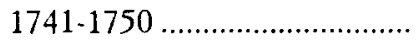 & 100 & 86 & 94 & 93 \\
\hline 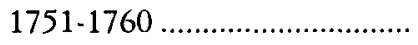 & 99 & 106 & 84 & 96 \\
\hline 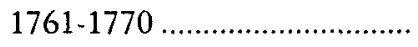 & 110 & 92 & 78 & 93 \\
\hline 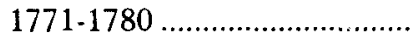 & 94 & 86 & 86 & 89 \\
\hline 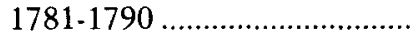 & 108 & 91 & 101 & 100 \\
\hline 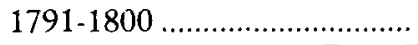 & 109 & 77 & 95 & 93 \\
\hline
\end{tabular}

Fuentes: Santiago de Chile: Larraín (1990). Potosí: Tandeter y Watchel (1992). Lima: Macera (1992). Sobre su elaboración ver texto. 


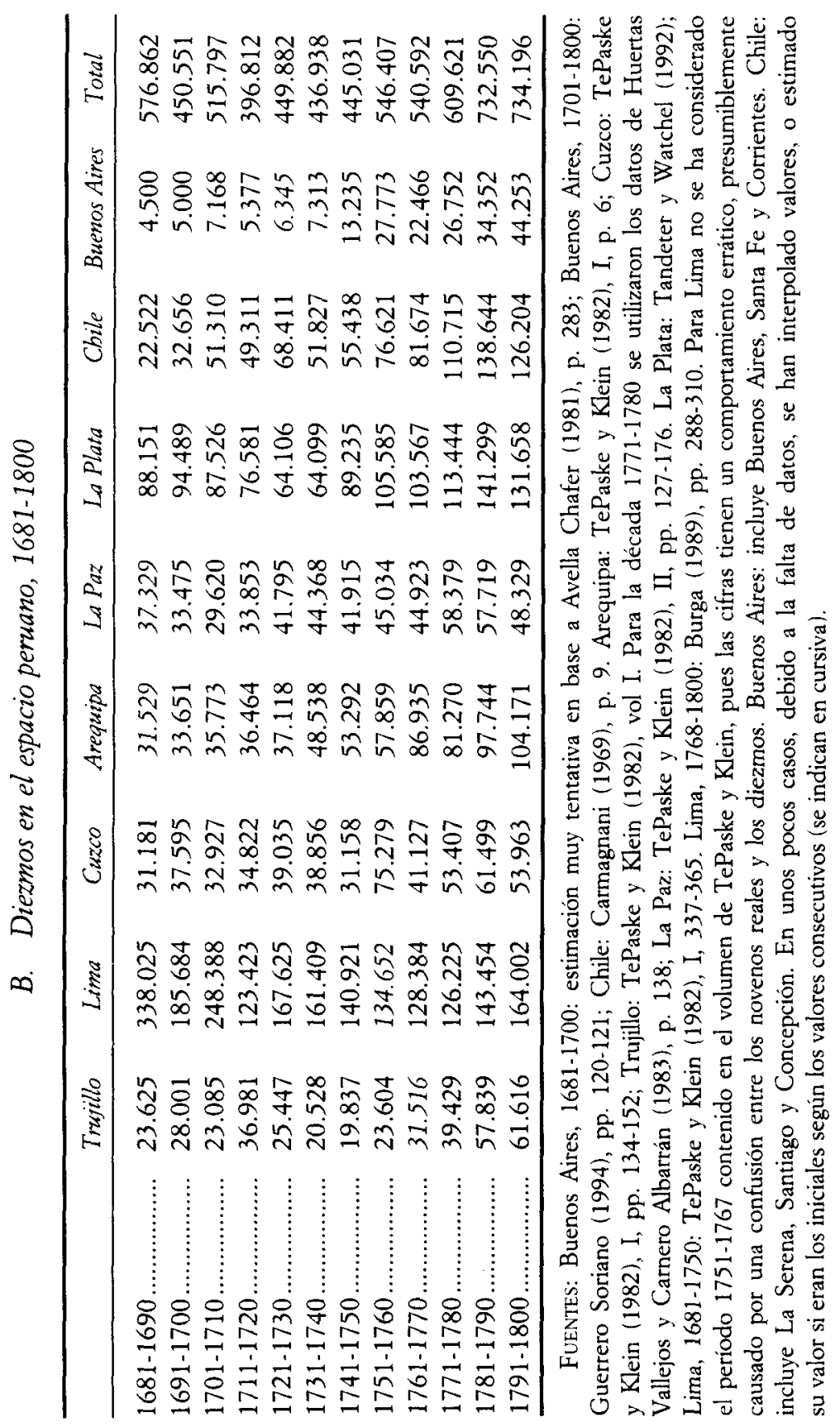

